Outcome Throughout May and June 2020, GOSH welcomed 65 Aspirant Nurses in total, 62 of whom had Junior Band 5 Staff Nurse conditional offers with us for September 2020. This resulted in us being able to supplement the workforce over the challenging months of the pandemic, while supporting a smooth transition into their Junior Band 5 Staff Nurse roles in the autumn.

Conclusion The benefits of this novel approach included:

- A smooth transition of Aspirant Nurses in to their next phase of employment as registered nurses

- Prevented disruption to the teams and rosters

- Supported continuity of care for patients

- Reduced the risk of attrition

- Having already completed induction, they did not require a supernumerary period on commencing in their Junior Band 5 Staff Nurse role

- Inclusion in workforce numbers throughout this period and immediately from when they commenced in post as Junior Band 5 Staff Nurses on the 28th September supported safe staffing and Care Hours Per Patient Day (CHPPD) data.

- Promoted GOSH as a good employer

- Reduced the impact on HR and payroll by reducing additional workload

\section{USING QUALITY IMPROVEMENT METHODOLOGY TO ELIMINATE HARM TO GOSH PATIENTS THROUGH STANDARDISED DEVICES AND ENHANCED RESOURCES}

Karleen Huggins, Nicola Wilson, Donna Wyan, Craig Knott, Helen Dunn, Johanna Andesson, Karen Ryan, Catherine Gee. Great Ormond Street Hospital

\subsection{6/archdischild-2020-gosh.96}

Background In 2019 GOSH identified variations in urethral catheter insertion and urinary catheter care. Datix highlighted this contributed to 28 potentially avoidable patient safety incidents in the previous 18 months. Lack of standardisation across the Trust was due to factors including; misbelief around female nurses catheterising males, patients having NG tubes inserted and multiple brands Trust-wide.

The aim Eliminate harm to ureteral catheterised patients.

Methods Supported by the Quality Improvement team, a Steering committee was formed from stakeholders Trust-wide. Reviewing incidents to identify themes and interventions, using the Model for Improvement (QI Tool), three work streams were established.

1. Standardised pathway for catheter care: Developing clinical practice guidelines for Urinary Catheterisation, standardising electronic documentation across the MDTs and introducing an escalation pathway.

2. Standardised trust training package: Quick reference posters, FAQs, Insertion videos and a Resources hub for quick/easy access. Facilitating 'Train the trainer study day' to improve overall nursing/medical knowledge.

3. Standardised devices: Standardised brands and devices then developing a device size reference guide.

The Project Plan kept focus on the aim and monitored time-dependant tasks.

Measurements; Catheter related incidences, Urology referrals for catheterisation (normal anatomy) and nursing staff knowledge on catheter cares.
Results Completed - Workstreams 1\&2, with a clear pathway, standardised electronic documentation, Trust-wide training package and a suite of resources. Workstream 3 still in progress, with an agreement to consolidate to two preferred devices

Discussion Our success is based on multidisciplinary working, using QI methodology and tools (Driver Diagrams) to identify primary drivers and simultaneously make decisions on how to improve care for catheterised patients.

*Covid has impacted results but initial findings support our prediction that access to resources increases knowledge and skill, resulting in an impact on incidents.

Conclusion Staff now have all the resources needed to provide excellent care for catheterised patients.

$*$ No ethical clearance needed

\section{PAEDIATRIC INTENSIVE CARE RETRIEVAL - FAMILIES' EXPERIENCE OF THEIR CHILD'S JOURNEY TO INTENSIVE CARE}

Ruth Evans, Victoria Barber, Padmanabhan Ramnarayan, Jo Wray. Great Ormond Street Hospital

10.1136/archdischild-2020-gosh.97

Background Each year, nearly 5000 critically ill children and young people (CYP) are transported by paediatric intensive care retrieval teams (PICRTs) from general hospitals to UK paediatric intensive care units (PICUs). Great Ormond Street Hospital (GOSH) accepts the greatest proportion of patients annual (circa 600) most retrieved by the GOSH based retrieval service CATS (Children's Acute Transport Service). Our study aimed to understand the experiences of families whose child used these services including those who were transferred using CATS.

Methods Parents of transported CYP were approached while in PICU and invited to participate in the study, which included filling out a questionnaire about their experience of their child's journey.

Results 24 (of 25) UK PICUs located in 21 NHS Trusts participated, 4558 CYP were identified as eligible for the study over a recruitment period of 12 consecutive months (January 2018-2019); 2838 consented (response rate of 62\%), 2133 completed questionnaires were received (73.5\% of consenting parents) and 1796 (84.2\%) questionnaires related to unique transfers by the 9 specialist PICRT in England and Wales, 16\% of responses were received from patients transferred into GOSH. Most families reported the overall experience and quality of care as 'excellent' ( $\mathrm{n}=1574 ; 87.6 \% ; \mathrm{n}=1615 ; 89.9 \%$ respectively) but a small proportion of families reported feeling confused about what was happening during transport $(n=193$; $10.7 \%)$, felt the transport team did not listen to them $(\mathrm{n}=27 ; 1.5 \%)$, and did not understand answers to questions asked $(\mathrm{n}=37 ; 2.1 \%)$.

Conclusions Service development is helped by understanding when and why experiences are sub-optimal and while quality of care delivered by PICRT appears to be almost universally perceived as 'excellent' families' experience is impacted by communication and could be improved through individualised -tailored- communication.

Acknowledgement this study was funded by the NIHR HS\&DR. 